\title{
Autoritäre Sexualpädagogik?
}

\section{Eine Analyse des Praxisbuches „Fit for Love“}

FERDINAND BACKÖFER

Die Debatten um (vermeintliche) Sexualpädagogik stellen eine zentrale Diskursarena für antifeministische Agitation und Interventionen dar (vgl. Oldemeier/Backöfer/Maurer/ Aleksin in diesem Band). Antifeministische Argumentationen plädieren in diesem Zusammenhang oft für ein alleiniges Erziehungsrecht der Eltern, das gegen die ,Indoktrination“ der (vorgeblich) institutionalisierten Sexualpädagogik verteidigt werden müsse (ebd.). Recherchen in diesem Feld zeigen aber, dass auch antifeministische Akteur_innen ${ }^{1}$ selbst sexualpädagogische Konzepte produzieren und diese - z.B. über Tagungen wie Wertevoll Aufklären im Jahr 2018 - in ihren Netzwerken verbreiten (Kemper 2018a).

Publikationen solcher Konzepte sind dabei überwiegend im gleichen Zeitraum zu verzeichnen, in dem die maßgeblichen Diskursereignisse der Kontroversen um (Sexual-)Pädagogik in der BRD stattgefunden haben (Oldemeier/Backöfer/Maurer/ Aleksin in diesem Band). So wurde 2014 etwa die Kampagne Prinzipen der Sexualpädagogik gestartet (Katholische Presseagentur 2014), von antifeministischen Akteur_innen wie Barbara Gerl-Falkovitz und Gerhardt Amendt gestaltet und unterzeichnet. Gerl-Falkovitz, die Hauptverantwortliche der Prinzipien, ist Leiterin des Studiengangs Entwicklungssensible Sexualpädagogik $($ der Hochschule Benedikt XVI. Heiligenkreuz (Initiative Christliche Familie 2018).

Das Label ,entwicklungssensibel' wird auch in anderen Veröffentlichungen aus diesem Feld aufgegriffen, so zum Beispiel in Powergirls \& Starke Kerle - 9 Unterrichtseinheiten für einen ganzheitlichen und entwicklungssensiblen Sexualkundeun-

1 Ich verwende den Unterstrich, auch Gender-Gap genannt, um damit eine Leerstelle bzw. einen Freiraum in Bezug auf Geschlecht zu markieren. So können nicht nur alle denkbaren Geschlechterpositionen, auch jenseits der Binarität von weiblich und männlich, adressiert werden, sondern auch die Versuche, der Kategorie Geschlecht zu entkommen. 
terricht von Regula Lehmann, Jakob Pastötter und Philip Pöschl aus dem Jahr 2018. Das Buch basiert höchstwahrscheinlich auf den beiden Publikationen Wir Powergirls und Rakete Startklar: Wie aus Jungs echte Kerle werden, bei denen Lehmann Co-Autorin ist und die im Jahr 2017 mit dem Deutschen Schulbuchpreis ausgezeichnet wurden (Kemper 2018b). Neben Pastötter ist auch Lehmann einschlägig im antifeministischen Diskurs positioniert: Eine von ihr mitgeführte Initiative von Sexualpädagog_innen ließ schon 2011 verlautbaren: „Nicht alle sexuellen Orientierungen und Lebensstile haben Anspruch auf gleiche Gewichtung“ (Schweizerische Interessengemeinschaft Sexualerziehung 2011: 1) und forderte: „[D]ie emanzipatorische Sexualpädagogik soll nicht Grundlage der Sexualkunde [...] sein.“ (Ebd.: 2) Mit dem Deutschen Schulbuchpreis werden, anders als der offiziell und neutral klingende Name suggeriert, laut den Förderkriterien Werke prämiert, „die Ehrfurcht vor Gott, Nächstenliebe, Toleranz und Dialogfähigkeit auf der Grundlage einer eigenen ethisch hohen, christlichen Überzeugung vermitteln“ (Schweidler o.J.).

Der christlich-konservative bis christlich-reaktionäre Hintergrund, der die hier angesprochenen sexualpädagogischen Konzepte eint, wird nicht unbedingt transparent gemacht. Begleitend zur Fallstudie zu (Sexual-)Pädagogik des REVERSE-Projekts wurde deshalb, angelehnt an die wissenschaftliche Methode der inhaltlich strukturierenden Dokumentenanalyse (Mayring 2002: 47), eine genauere Untersuchung prominenter Konzepte und Projekte aus diesem Umfeld vorgenommen. Der Fokus lag dabei auf dem Aspekt der Quellenkritik und einer Interpretation im Lichte relevanter antifeministischer Diskurselemente.

Explizit ausgewählt wurde dafür u.a. das ,Praxisbuch“ Fit for Love von Tabea Freitag (2015).

\section{QUELLENKRITIK}

\subsection{Beschreibung, Relevanz und Kontextualisierung des Materials}

Fit for Love ist nach der eigenen Selbstbeschreibung ein „Praxisbuch zur Prävention von Internet-Pornografie-Konsum“ im Sinne einer ,bindungsorientierte[n] Sexualpädagogik“. Der einführende ,wissenschaftliche Teil‘ (Freitag 2015: 16-57) begründet den folgenden ,pädagogischen Teil', kritisiert zugleich eine vermeintliche ,Sexualpädagogik der Vielfalt‘ und resümiert Wirkungsforschung zu Pornografie. Der sehr ausführliche ,pädagogische Teil‘ (ebd.: 58-173) ist gegliedert nach Themen- bzw. Arbeitseinheiten, die für eine Zielgruppe von 13- bis 19-Jährigen konzipiert wurden. Jede Arbeitseinheit ist mit Vorschlägen für Methoden versehen, über die die zentralen Inhalte vermittelt werden sollen. Das Buch enthält überdies exemplarische Arbeitsblätter und ,Stundenentwürfe‘. Dieses quasi didaktisch aufbe- 
reitete Angebot macht vermutlich die Attraktivität des ,Praxisbuchs' aus, das mittlerweile drei Mal aufgelegt worden ist (erstmals 2013, das dritte Mal 2015 - mit einer Gesamtauflage von 4.500 Exemplaren).

Das Buch von Tabea Freitag hat 2013 den Gesundheitspreis der BARMER GEK gewonnen. Die von der Autorin und ihrem Ehemann Eberhard Freitag geführte return: Fachstelle Mediensucht, die auch Fortbildungen und ,Bildungstage' anbietet, wurde bis 2016 von der Stadt Hannover gefördert; mittlerweile erfolgt eine Förderung durch die Region Hannover. Die Fachstelle Mediensucht erscheint in Beiträgen von Zeitungen (wie BILD, Mindener Tageblatt, Wallstreet Online) und Fernsehen (ZDF Info, NDR) (Return 2019). Der hier analysierten Veröffentlichung kann vor diesem Hintergrund ein gewisser Impact sowie ein breiter anschlussfähiges ,respektables Image‘ zugerechnet werden.

Zugleich wird das Buch von Antifeminist_innen empfohlen; Hedwig von Beverfoerde, die führende Protagonistin der Demo für alle, weist auf ihrer Seite Elternaktion auf die Fachstelle hin, und eine Empfehlung des Buches erfolgt durch Jakob Pastötter, der die Vorworte zur Publikation verfasst hat. Pastötter selbst ist Mitautor des bereits erwähnten Buches Powergirls \& Starke Kerle sowie Mitunterzeichner der ebenfalls bereits angeführten Prinzipien der Sexualpädagogik (GerlFalkovitz 2014). Es ist genau diese Schnittstelle aus Anschlussfähigkeit für eine breitere Öffentlichkeit und Verbindungen zum organisierten Antifeminismus, die Fit for Love so relevant für eine Untersuchung macht - vor allem, weil eine genaue Betrachtung der Vorgehensweise in diesem Buch exemplarisch aufzeigt, welche Strategie christliche Antifeminismen aktuell zu verfolgen scheinen und wie erfolgreich sie damit sein können.

\subsection{Autorin und Selbstverortung des Werkes}

Tabea Freitag ist Mitglied des Forums Deutscher Katholiken (Forum Deutscher Katholiken e.V. o.J. a), einer papsttreuen katholischen Organisation (Forum Deutscher Katholiken e.V. o.J. b). Das Forum lehnt Homosexualität, Sex vor der Ehe sowie Scheidungen ab und wird selbst von konservativen katholischen Wissenschaftler_innen als stark wertekonservativ, mit offenen Flanken und expliziten Verbindungen zum Rechtspopulismus eingeordnet (Meinhardt 2017). Trotz der Verbindungen des Forums zu (rechts-)konservativen christlichen Kreisen findet sich in Fit for Love keine einzige explizit konfessionelle Argumentation. Freitag begründet aber ausführlich, dass es eine wertneutrale Position ohne politische oder weltanschauliche Elemente gar nicht geben könne, weswegen sie ankündigt, ihre Grundlagen und Denkvoraussetzungen offenzulegen (Freitag 2015: 16). Diese sind nach eigener Aussage ,Wissenschaftlichkeit' (ebd.) und die - laut Grundgesetz zu achtende und zu wahrende -, Würde des Menschen“ (Freitag 2015: 22). Freitags religiöser 
Hintergrund und die Werte, die sie als Mitglied des Forums mitträgt, werden nicht erörtert. Dies legt die Lesart nahe, dass die Autorin diesen Hintergrund als ,Privatangelegenheit' betrachtet, die ihre inhaltlichen Ausführungen und ihren pädagogischen Ansatz unberührt lässt. Eine weiterführende Analyse zeigt allerdings, dass dies nicht plausibel ist.

\section{INHALTLICHE KRITIK}

\subsection{Wissenschaftlichkeit}

Freitag ist deutlich darum bemüht ihr Werk als wissenschaftlich fundiert darzustellen. Ihr Literaturverzeichnis ist sehr ausführlich, und sie betont selbst immer wieder wissenschaftlich notwendige Differenzierungen, etwa im Hinblick auf die Unterscheidung zwischen Korrelation und Kausalität (Freitag 2015: 36). Gleichzeitig unterläuft ihr aber selbst eben jene Verwischung: Sie zitiert zwei Studien (Bersamin et al. 2014 sowie Långström/Hanson 2006), um ihre Behauptung zu stützen, dass Promiskuität ,nachweislich schädliche Folgen“ (Freitag 2015: 20) habe (Behauptung einer Kausalität), obwohl beide Studien nur einen Zusammenhang (Korrelation) belegen können.

Die von ihr referierten Befunde aus der Wirkungsforschung zu PornografieKonsum sind ebenfalls nicht so eindeutig, wie behauptet: Freitags Darstellung folgt hier meist Annahmen aus der Medienforschung, die von einer mehr oder weniger direkten Beeinflussung und Prägung durch Medien ausgehen, was im aktuellen wissenschaftlichen Diskurs umstritten ist (Vollbrecht 2010: 146). Weller (2011) kritisiert die mangelnde Validität bzw. den mangelnden Wirklichkeitsbezug der von Freitag hauptsächlich genutzten Studien, die sich lediglich auf Laborexperimente beziehen.

„Andere (Fragebogen-)Studien, die realen Pornokonsum erfassen und in Bezug zu sexuellen Einstellungen oder Verhaltensweisen setzen, konstatieren i.d.R. Zusammenhänge, die aber keine kausale Interpretation erlauben (Schmidt 2009). Für nachhaltige Verhaltenswirksamkeit [...] gibt es - abgesehen von kriminologischen und klinischen Einzelfällen - keine Belege.“ (Weller 2011: 9)

$\mathrm{Zu}$ Freitags zentraler These, dass Pornos die Fähigkeit zu Intimität und Beziehungen zerstören und Sexualität ,entleeren“ (Freitag 2015: 37), gibt es ebenfalls durchaus anders lautende Befunde. So fand Vollbrecht (2010: 161) in seiner systematischen Sichtung qualitativer Studien z.B. keinen signifikanten Zusammenhang. Dass ein Ergebnis ,signifikant“ ist, bedeutet, dass unabhängig vom Zufall ein Zusam- 
menhang nachgewiesen werden konnte. Das ist einem nicht-akademischen Publikum oft nicht bewusst. Dieser Umstand trägt dazu bei, dass Freitag Pornografie als hoch gefährlich darstellen kann; so etwa, wenn sie schreibt, Pornografie-Konsum sei ein „signifikanter Prädikator für sexuelle Aggression“ (Freitag 2015: 44). Effektstärken, als für die Wirkungsforschung relevanteste Messgröße, werden von ihr aber außer Acht gelassen. Diese würden ihre Darstellung auch stark relativieren: So spricht kriminologische Forschung im Hinblick auf ,Pornografie-Konsum und Gewalt' zwar durchaus von signifikanten Zusammenhängen, aber von - im Vergleich mit anderen Faktoren - nur geringen Effektstärken (Hill 2018).

$\mathrm{Zu}$ guter Letzt irritiert, dass die Publikation einer Autorin, die eine Fachstelle für Mediensucht leitet, an keiner Stelle eine wissenschaftliche Definition von Sucht einführt oder Kriterien dafür aufstellt, wie Sucht zu erkennen und von anderen Konsumprofilen zu unterscheiden sei. Wenn Freitag schreibt, dass sie Jugendliche bestimmt sieht von ,drängenden konditionierten Dopaminschüben [...], die sie an die Gier nach dem nächsten Kick am Bildschirm bindet [sic!]“ (Freitag 2015: 51), offenbart sie ein Suchtverständnis, das nicht über einen schlichten Behaviorismus hinaus geht. Ein solcher gilt in der aktuellen Medien- und Suchtforschung aber als verkürzt (Vollbrecht 2010: 146; Degkwitz 2007: 68ff).

Die von Freitag präsentierten wissenschaftlichen Befunde sind also mindestens streitbar, wirken durch fehlende Kontextualisierung tendenziös oder werden schlicht falsch wiedergegeben. Es scheint hier eher darum zu gehen, eine reflektierte und fundierte wissenschaftliche Autorität $\mathrm{zu}$ behaupten, um dadurch in nichtwissenschaftliche Diskurse hinein zu wirken (Näser-Lather in diesem Band). Die von Freitag mit solchem Gestus proklamierten Thesen sind inhaltlich aus dem populärwissenschaftlichen Diskurs der Massenmedien unter dem Schlagwort, Sexuelle Verwahrlosung' bekannt. Freitag wiederholt lediglich die dort vorgetragene Befürchtung: ,Jungen und Mädchen entwickelten dadurch [durch erhöhten Pornografie-Konsum] ein zunehmend instrumentelles Verhältnis zur Sexualität und zu ihren Partnern bzw. Partnerinnen und eine egoistische Bedürfnisbefriedigung löse somit sexuelle Beziehungen, die auf Liebe und Treue basieren, ab.“ (Klein 2010: 167) Kritische Sexualwissenschaftler_innen haben schon vor Jahren gegen die wissenschaftlich äußerst fragwürdige Skandalisierung einer vermeintlichen ,Sexuellen Verwahrlosung der Jugend' argumentiert (Weller 2011: 8f).

Das Besondere an Freitags Publikation ist deshalb die Rahmung der Skandalisierung von Pornografie. Denn Freitag führt die von ihr konstatierten Defizite und Gefahren nicht einfach, kulturpessimistisch, auf die Jugendlichen oder, alternativ, die (neuen) Medien zurück (Schmidt 2009). Bei ihr sind ,die Schuldigen“ vielmehr ,die herrschende Sexualpädagogik‘ und -wissenschaft. Denn, so Freitag, 
lem sind die Erwachsenen, die ihre Verantwortung dafür leugnen, der nächsten Generation in immer jüngerem Alter und immer härterer Dosis in bald jedem Lebensbereich [...] die Produkte einer zutiefst menschenverachtenden ,Nimm dir, was du willst'-Philosophie und Industrie zuzumuten und ihnen dadurch ein verarmtes, kaltes und zutiefst unmenschliches Bild von Sexualität überstülpen“ (Freitag 2015: 51).

\subsection{Dezidiertes Gegenmodell zur ,Sexualpädagogik der Vielfalt‘}

Fit for Love ordnet sich an mehreren Stellen explizit in den Diskurs um (Sexual-) Pädagogik im deutschsprachigen Raum ein, was in den Vorworten von Jakob Pastötter besonders deutlich wird. So schreibt er in seinem Vorwort zur zweiten Auflage:

„Weiterhin wird verstärkt politisch und institutionell Druck gemacht, eine überall wahrgenommene ,diskriminierende Heteronormativität ‘ durch eine ,Sexualpädagogik der Vielfalt‘ zu bekämpfen [...] Dass ein Großteil der Öffentlichkeit einer solchen Zwangsbeglückung der Kinder und Jugendlichen in Kitas und Schulen skeptisch gegenübersteht, wird nur als Beweis für eine als schädlich für eine freie Sexualentwicklung angesehene, in einem Status Quo verhaftete Einstellung gewertet, die es über die Köpfe der Eltern hinweg zu überwinden gelte. [...] Die erste Auflage von ,Fit for Love?` hatte einen ganz wesentlichen Anteil daran, dass endlich ein Diskurs angestoßen wurde.“ (Pastötter 2014: 9).

Schon hier werden antifeministische Motive, wie das einer drohenden Indoktrination, wiederholt. Auch Freitag selbst widmet mehrere Seiten der Kritik einer ,Sexualpädagogik der Vielfalt‘ (Freitag 2015: 23-36). Dabei werden nicht nur das konkrete Konzept der Sexualpädagogik der Vielfalt, sondern auch andere Ansätze der Sexualpädagogik sowie queere Antidiskriminierungsprojekte kritisiert. Immer wieder scheinen gängige Topoi des antifeministischen Diskurses auf: So wiederholt Freitag die Rede von der „Genderideologie“, die von kleinen Lobbygruppen „ohne demokratische Legitimation“ durchgesetzt werden solle; diese hätten ,eine Art Meinungsmonopol“" (Freitag 2015: 27). ,Gender' sei keine Theorie, sondern Begleitideologie eines politischen Programms. Diese Argumentation erinnert an die im antifeministischen Diskurs verbreiteten Verschwörungsideologien; sie wird lediglich mit der (nichtwissenschaftlichen) Publikation eines weiteren Forum-Mitglieds belegt. Zwar räumt Freitag ein, dass Antidiskriminierung ein wichtiges Thema sei (Freitag 2015: 34), ignoriert aber implizit immer wieder sexuelle Orientierungen und Geschlechtsidentitäten abseits der Heteronormativität. Wenn Homo- und BiSexualität besprochen werden, erscheinen sie durch den jeweiligen Kontext implizit als Bedrohung. Hier ein Beispiel: 


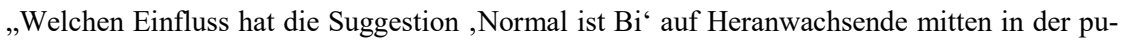
bertären Phase der hormonellen Veränderungen, Verunsicherung, Normorientierung und sexuellen Identitätsfindung? [...] Das entstehende emotionale Beziehungschaos gleichzeitiger oder wechselnder Verliebtheit in beide Geschlechter dürfte die Bewältigung dieser ohnehin schon herausfordernden Entwicklungsphase nicht erleichtern.“ (Ebd.: 32)

Danach zitiert Freitag unkommentiert Studien, die ein Anwachsen von bi-sexuellen Einstellungen und Erfahrungen bei Jugendlichen belegen und beendet damit die Ausführung. Hier wird die Argumentationsweise des Werkes deutlich, die als ,subtil-suggestiv“ charakterisiert werden kann: Keiner der üblichen Vorwürfe des antifeministischen Diskurses wird von Freitag selbst explizit geäußert. Diese werden lediglich , angespielt ${ }^{\text {- }}$ durch rhetorische Fragen und den spezifischen Kontext der Darstellung von Forschungsergebnissen. Es bleibt dem_der Leser_in überlassen, die implizite Bedeutung des Formulierten (hier: ,Homosexualität verwirrt', ,Steigende homo- und bi-sexuelle Erfahrungen sind besorgniserregend“ und ,Sexualpädagogik kann die sexuelle Orientierung von Jugendlichen direkt beeinflussen') selbst herzustellen. Der Autorin wiederum bleibt die Möglichkeit, jeden kritischen Einwand mit dem Verweis auf die manifesten Inhalte ihres Textes von sich zu weisen. Ein solches Vorgehen der „Insinuation“ ist mittlerweile vielfach als Diskursstrategie der neuen Rechten beschrieben worden (Bruns/Strobl 2015: 209). Allerdings hält Freitag diese Subtilität nicht immer aufrecht. So spricht sie auf Seite 31 offen von einem „Meinungskartell“ und wirft die Frage auf, „ob Lobbygruppen unter dem Deckmantel der Antidiskriminierung Kinder und Jugendliche in einem ganz zentralen Aspekt ihrer Persönlichkeitsentwicklung indoktrinieren und ihre Intuitionen verwirren und verstören dürfen“" (Freitag 2015: 31).

Überdeutlich wird die Ausrichtung von Autorin und Werk dann dort, wo die wissenschaftliche Form verlassen wird, um praktisch zu werden: in den pädagogischen Arbeitseinheiten.

\subsection{Rigide Normorientierung und fragwürdige Didaktik}

In Freitags pädagogischen Ausführungen wird ihre Vorstellung von Sexualität und auch von Pädagogik - klar erkennbar. Den Jugendlichen soll z.B. vorgetragen werden: „Es geht ja um zwei Menschen und deren größtmögliche Nähe. Da passt kein Blatt zwischen. Sexualität ist im Kern die intime Begegnung zwischen zwei Menschen. Sie gehen eine Verbindung ein, aus der zudem neues Leben, ein Mensch entstehen kann." (Freitag 2015: 144) Hier wie an vielen anderen Stellen wird das dem Text zugrunde gelegte Bild von ,richtiger' Sexualität (die nur in einer monogamen, heterosexuellen Partnerschaft mit geteilter Zeugungsfähigkeit möglich erscheint) explizit. Welche Rolle Treue und Enthaltsamkeit für Freitag dabei spielen 
und welche Mittel sie vorschlägt, um diese Werte an Jugendliche zu ,vermitteln', zeigen dabei andere Einheiten:

Auf Seite 140 wird eine Übung vorgeschlagen, bei der anhand eines Klebebands, das nach mehrmaligem Ankleben auf die Haut an Klebekraft verliert, demonstriert werden soll, wie sich menschliche Bindungsfähigkeit auch ,verbraucht ${ }^{6}$ und pro eingegangener Beziehung schwächer wird. Diese und andere inhaltlich höchst fragwürdigen und zudem stark suggestiven Darstellungen durchziehen Freitags Didaktik und treten besonders offen zu Tage auf Seite 128: Hier werden Jugendliche mit Suggestivfragen konfrontiert, wie z.B.:

„Wie wirkt es sich auf die spätere Partnerschaft aus, wenn ich vorher ,alles ausprobiert ' habe? Wird mir der Partner bzw. die Partnerin vertrauen? Wie würde es mir selbst gehen, wenn mein Partner bzw. meine Partnerin viele sexuelle Beziehungen vor mir hatte? Fühle ich mich noch einzigartig [...]? Hätte ich eher Angst, nicht zu genügen?“ (Ebd.)

Diese Fragen transportieren, eindeutig richtige' Normen und die pädagogische Einheit sieht nicht vor, diese zu diskutieren. Stattdessen werden sie den Jugendlichen durch suggestiv verstärkte oder sogar erst erzeugte Ängste und Verunsicherungen quasi ,eingebläut'.

Das dabei mitschwingende Moment der Beschämung in dieser Art von Didaktik wird in der ,Apfelbiss-Methode " noch auf die Spitze getrieben: Die Jugendlichen sollen einen Apfel herumgeben und jeweils von ihm abbeißen, solange, bis sich eine Person weigert davon zu essen, weil der Apfel zu ,abgegessen' und damit ,ekelhaft' geworden ist. ${ }^{2}$ Wie diese metaphorische Gleichsetzung von Menschen, die (seriell) wechselnde Sexualpartner_innen haben mit unappetitlichem Obst mit Freitags Selbstverpflichtung auf die Wahrung von Menschenwürde vereinbar sein soll, erschließt sich nicht.

Gerade im methodisch-praktischen Teil des Buches wird sehr deutlich, dass Fit for Love im Kern eine autoritäre Erziehung zur (heterosexuellen) Keuschheit leisten will, die Sex außerhalb heterosexueller Paarbeziehungen (besser: Ehen, noch besser: Familien) verdammt und dafür auf eine Didaktik zurückgreift, die mit Verunsicherung, Verängstigung und Beschämung arbeitet.

2 Diese Methode wird als von TeenSTAR übernommen angegeben. TeenSTAR ist ein Verein, der auch ,Bildungstage ' durchführt und in Österreich und Deutschland aktiv ist. In Österreich wurde dem Verein vorerst untersagt, weiter an Schulen zu arbeiten, da er in der Kritik stand, christlich-fundamentalistische Inhalte zu vertreten und „,[h]omophobe Propaganda“ zu betreiben (Austrian Presse Agentur 2018). 


\section{FAZIT}

Die Analyse von Tabea Freitags Fit for Love zeigt, wie subtil-suggestiv in der Rhetorik, aber auch offen-suggestiv im praktischen Tun autoritäre ,Gegenmodelle ‘ der Sexualpädagogik von antifeministischen Akteur_innen arbeiten. Freitags recht eindeutige Positionierung in einem antifeministischen Diskurs- und Akteur_innenfeld und ihre fragwürdige Darstellung wissenschaftlicher Ergebnisse sind für Leser_innen ohne differenziertere Vorkenntnisse in beiden Bereichen nur schwer zu erkennen und noch schwerer zu kritisieren. Freitags Zusammenarbeit mit Pastötter lässt neben ihrer Mitgliedschaft im Forum deutscher Katholiken - auch bei ihr selbst einen entsprechenden ideologischen Hintergrund vermuten, den sie aber im ganzen Buch nie transparent werden lässt. Zugleich zeigt die Betrachtung der von ihr vorgeschlagenen Arbeitseinheiten und Methoden, dass ihr Ansatz die Werte des Forums mindestens in Bezug auf den Aspekt der Treue bzw. Keuschheit klar verfolgt.

Nur durch Freitags Verschleierung dieser Hintergründe lässt sich wohl die relative Akzeptanz und der Erfolg ihres Werkes erklären (man denke an die Auszeichnung durch eine gesetzliche Krankenkasse!), das im Kern fachliche und wissenschaftliche Mängel aufweist, didaktisch fragwürdig vorgeht und höchst wahrscheinlich politisch-ideologisch motiviert ist, ohne dies transparent zu machen.

Dies lässt die Vermutung plausibel erscheinen, dass antifeministische Akteur_innen einen bewussten und gezielten Kampf im Feld der Pädagogik austragen und ihre politischen Ziele auch dort verwirklichen wollen. Dass dafür wissenschaftliche und demokratische Diskurse instrumentalisiert werden, ist auch aus anderen Feldern des antifeministischen Diskurses bekannt. Publikationen wie Fit for Love oder Initiativen wie der Deutsche Schulbuchpreis zeigen dabei aber, wie wachsam eine demokratische Öffentlichkeit gegenüber autoritären Sexualpädagogiken sein muss, wenn sie diese nicht unwissentlich akzeptieren oder sogar fördern will.

\section{LITERATUR}

Bersamin, Melina M./Zamboanga, Byron L./Schwartz, Seth J./Donnellan, M. Brent/ Hudson, Monika/Weisskirch, Robert S./Kim, Su Yeong/Agocha, V. Bede/Whitbourne, Susan Krauss/Caraway, S. Jean (2014): „Risky Business: Is There an Association between Casual Sex and Mental Health among Emerging Adults?“, in: The Journal of Sex Research 51 (1), S. 43-51. [DOI: https://doi.org/10. 1080/00224499.2013.772088].

Bruns, Julian/Strobl, Natascha (2015): „(Anti-)Emanzipatorische Antworten von Rechts“, in: Zeitschrift für Sozialen Fortschritt 4 (4), S. 205-217. 
Degkwitz, Peter (2007): „Plädoyer für ein psychosoziales Verständnis von Sucht“, in: Bernd Dollinger/Henning Schmidt-Semisch (Hg.), Sozialwissenschaftliche Suchtforschung, Wiesbaden: Springer VS, S. 59-81.

Henninger, Annette/Birsl, Ursula (Hg.) (2020): Antifeminismen. ,Krisen“-Diskurse mit gesellschaftsspaltendem Potential?, Bielefeld: transcipt.

Hill, Andreas (2018): „Wirkung von und Umgang mit Pornographie im Straf- und Massregelvollzug“. Vortrag gehalten bei der 4. Forensischen Fachtagung: Sexualität in der Forensik Psychiatrische Klinik Münsterlingen, 15.3.2018. [Präsentation unter https://t1p.de/vwt9; abgerufen am 28.10.19].

Klein, Alexandra (2010): „Jugend, Medien und Pornographie“, in: Schetsche/ Schmidt, Sexuelle Verwahrlosung. Empirische Befunde - Gesellschaftliche Diskurse - Sozialethische Reflexionen, S. 167-183.

Långström, Niklas/Hanson, R. Karl (2006): „High Rates of Sexual Behavior in the General Population: Correlates and Predictors“, in: Archives of Sexual Behaviour 35, S. 37-52. [DOI: https://doi.org/10.1007/s10508-006-8993-y].

Mayring, Philipp (2002): Einführung in die qualitative Sozialforschung. Eine Anleitung zu qualitativem Denken, Weinheim/Basel: Beltz.

Näser-Lather, Marion (2020): „Wissenschaftler_innen vs. Gender Studies. Argumentationen, Wirkungen und Kontexte einer, wissenschafts'-politischen Debatte“, in: Henninger/Birsl, Antifeminismen. ,Krisen“-Diskurse mit gesellschaftsspaltendem Potential?

Oldemeier, Anna Lena/Backöfer, Ferdinand/Maurer, Susanne/Aleksin, Katharina (2020): „Divergenz, Ambivalenz, Kongruenz. Verhältnisbestimmungen zwischen antifeministischem Diskurs und pädagogischem Feld“, in: Henninger/ Birsl, Antifeminismen. ,Krisen'-Diskurse mit gesellschaftsspaltendem Potential?

Schetsche, Michael/Schmidt, Renate-Berenike (Hg.) (2010): Sexuelle Verwahrlosung. Empirische Befunde - Gesellschaftliche Diskurse - Sozialethische Reflexionen, Wiesbaden: Springer VS.

Schmidt, Gunter (2009): „Fantasien der Jungen, Phantasmen der Alten“, in: BZgA Forum 1/2009, S. 27-32.

Vollbrecht, Ralf (2010): „Wirkung pornographischer Mediendarstellungen“, in: Schetsche/Schmidt, Sexuelle Verwahrlosung. Empirische Befunde - Gesellschaftliche Diskurse - Sozialethische Reflexionen, S. 145-165.

Weller, Konrad (2011): „Jugendsexualität und Medien“, in: KJug 56 (1), S. 8-12. 


\section{QUELLEN}

\section{Textkorpus}

Freitag, Tabea (2015): ,Fit for Love' - Praxisbuch zur Prävention von InternetPornografie-Konsum. 3. Aufl., Hannover: return Fachstelle Mediensucht.

Pastötter, Jakob (2014): „Vorwort zur zweiten Auflage“, in: Tabea Freitag,Fit for Love - Praxisbuch zur Prävention von Internet-Pornografie-Konsum. 3. Aufl., Hannover: return Fachstelle Mediensucht, S. 9.

\section{Weitere Quellen}

Austrian Presse Agentur (2018): Sexualkundeverein Teenstar wird vorerst aus Schulen verbannt. [https://www.derstandard.at/story/2000091902029/sexualkun deverein-teenstar-wird-aus-schulen-verbannt; abgerufen am 06.11.19].

Forum Deutscher Katholiken e.V. (o.J. a): Tabea Freitag. [http://forum-deutscherkatholiken.de/tabea-freitag/; abgerufen am 28.10.19].

Forum Deutscher Katholiken e.V. (o.J. b): Die Botschaft des Forums deutscher Katholiken. [http://forum-deutscher-katholiken.de/ueber-uns/die-botschaft-des-fo rums-deutscher-katholiken/; abgerufen am 28.10.19].

Gerl-Falkovitz, Hanna-Barbara (2014): Prinzipien Sexualpädagogik. Unterzeichner. [https://www.prinzipien-sexualpaedagogik.org/deutsch/unterzeichner/; abgerufen am 28.10.19].

Initiative Christliche Familie (2018): Entwicklungssensible Sexualpädagogik. [http://leib-bindung-identitaet.org; abgerufen am 28.10.19].

Katholische Presseagentur (2014): Fachleute empfehlen neue Wege in der Sexualpädagogik. [http://www.kath.net/news/48299; abgerufen am 28.10.19].

Kemper, Andreas (2018a): Wertevoll aufklären. [http://www.diskursatlas.de/index. php?title=Wertevoll_aufklären; abgerufen am 28.10.19].

Kemper, Andreas (2018b): Regula Lehmann. [http://www.diskursatlas.de/index. php?title=Regula_Lehmann; abgerufen am 28.10.19].

Meinhardt, Thomas (2017): „Radikalisierungstendenzen am rechten Rand der Kirche. Sind Katholiken anfällig für Rechtspopulismus?“ Originalinterview in Langfassung. Die gekürzte Fassung erschien in: Zeitschrift Franziskaner, Sommer 2017. [https://franziskaner.net/radikalisierungstendenzen/; abgerufen am 28.10.19].

Return gemeinnützige GmbH (2019): Was andere über uns berichten. [https://www. return-mediensucht.de/fachstelle/medienecho/; abgerufen am 28.10.19].

Schweidler, Walter (o.J.): Kriterien. [http://www.schulbuchpreis.de/kriterien.html; abgerufen am 28.10.19].

Schweizerische Interessengemeinschaft Sexualerziehung (2011): Forderungen betreffend Sexualunterricht. [https://www.human-life.ch/upload/dokumente/2011 
0225_IG-Serz_Grundlage-Forderungen_Sexualunterricht_mit-Fussn_v16.0HLI.pdf; abgerufen am 28.10.19]. 